\title{
The inverse kinematics analysis and simulation study on the controllable loading mechanism
}

\author{
Lin Zhang ${ }^{1, *}$, Cheng-ge $\mathrm{Wu}^{1}$, and Gan-wei $\mathrm{Cai}^{2}$ \\ ${ }^{1}$ Department of Engineering Machinery, Shandong Jiaotong University, Jinan 250357, China \\ ${ }^{2}$ Department of Mechanical Engineering, Guangxi University, Nanning 530004, China
}

\begin{abstract}
The multi-DOF controllable loading mechanism adopts the electric drive system to replace the hydraulic drive system of traditional loader, not only has higher transmission efficiency and has better mechanical and electrical integration level, but also overcome the shortcomings of high cost of manufacture and maintenance. The inverse kinematics model of this type of loading mechanism was built by means of closed loop vector method. According to the performance index of the loading mechanism, the inverse kinematics model was solved by means of MATLAB software, and the input motion law of the two driving lever at different period of time is obtained, and a small prototype is built, which provides the basis for control system programming of this new type of leading mechanism. To some extent, this research lays a foundation for further development of this type of loading mechanism.
\end{abstract}

\section{Introduction}

Because of the use of hydraulic transmission, the current loader's working device has many problems, such as low transmission efficiency, high failure rate of hydraulic system and high energy consumption [1-3]. In order to find ways to overcome these problems, under the funding of the doctoral scientific research foundation of Shandong Jiaotong University, the latest theory of mechanism [4-10] was applied to design the working devices of the construction machinery, and some of multi-DOF controllable loading mechanism was invented [11-12], which are mechanical loaders that can realize flexible control of bucket trajectory, and use link mechanism instead of hydraulic transmission. This kind of loading mechanism not only low in energy consumption, high in reliability and flexible in action, but also in full electric control, which is very suitable for the manufacture of a super small loader.

Multi-DOF controllable loading mechanism are common drive by multiple motors mounted on the frame. Therefore, through the kinematic analysis to obtaining the motion rule of the driving lever are the primary problems for the control of the controllable loading mechanism. The kinematic analysis methods of planar linkage mechanism include graphic method and analytic method [13-14], for the multi-bar linkage mechanism, kinematic analysis is often carried out with the aid of virtual prototyping technology [15-16]. In order to obtain the accurate parameter relationship between motion input and output of the

\footnotetext{
* Corresponding author: wolfunion@163.com
} 
controllable loading mechanism, this paper will use the closed loop vector method to establish an inverse kinematics model of a nine bar two DOF controllable loading mechanism, and to solve this model with the help of MATLAB numerical calculation tools. These studies will provide a reference for further research and application of this type of controllable loading mechanism.

\section{Analysis of controllable loading mechanism}

The basic structure of the two degree of freedom controllable loader is shown in figure 1 . The first driving lever controls the lifting of the boom, and the second driving lever controls the turning of the bucket. Through the programmable of the controllable motor, the coupling motion of the controllable loading mechanism is realized, and so as to realize the loading and unloading operation.

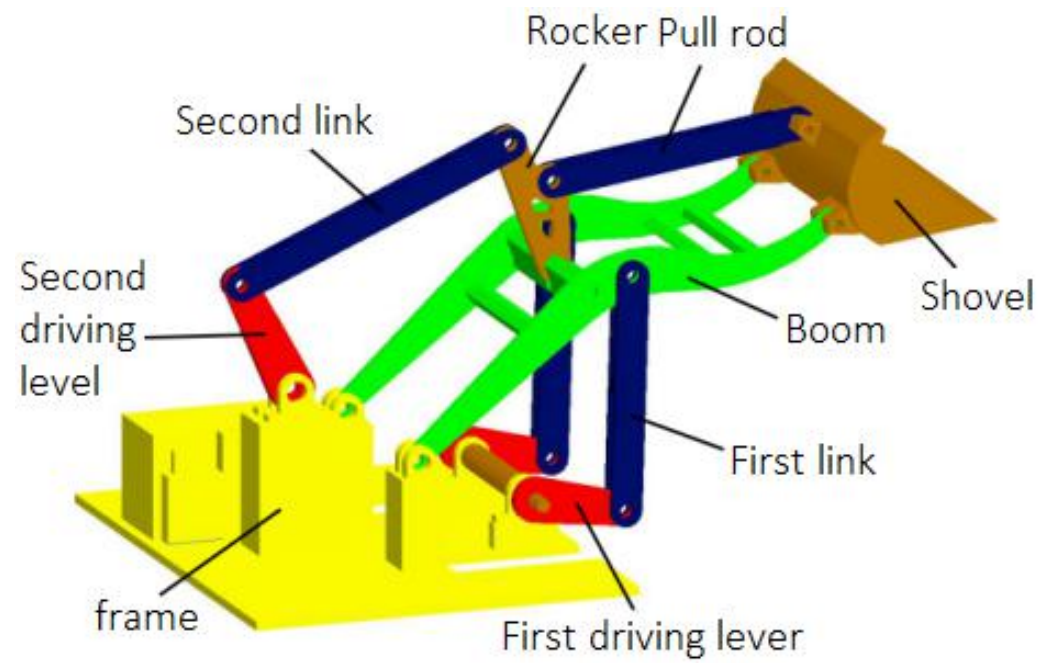

Fig. 1. The basic structure of the controllable loader.

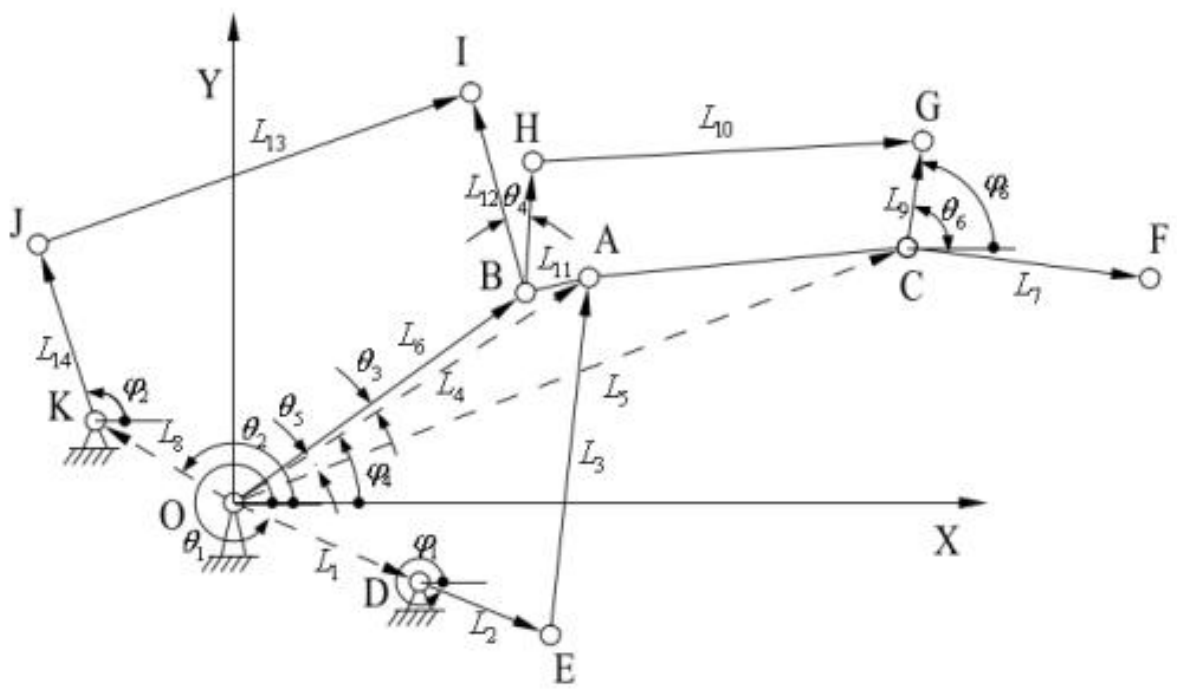

Fig. 2. The kinematic diagram of controllable loading mechanism. 


\section{Inverse kinematics analysis}

Controllable loading mechanism kinematic diagram as shown in figure 2, each component vector in the mechanism is represented by $L_{i}(i=1,2, \ldots, 14)$, the length of the component is represented by $l_{i}$, the position angles of the vector $L_{i}$ are represented by $\theta_{1}, \varphi_{1}, \varphi_{3}, \varphi_{4}, \varphi_{10}, \varphi_{5}, \varphi_{12}, \theta_{2}, \varphi_{6}, \varphi_{7}, \varphi_{8}, \varphi_{9}, \varphi_{11}, \varphi_{2}$. The inverse kinematics problem of this type of working device can be described as: the structural parameters in the working device are known, and the motion rules and angular displacement of the boom and the bucket are known, Solving the motion law of two driving level, including angular displacement $\varphi_{1}, \varphi_{2}$ and angular velocity $\omega_{1}, \omega_{2}$.

The closed loops ODEA、OBHGC、OBIJK vector equation is established respectively. By introducing the complex number and Euler inequality, the equation can be obtained:

$$
\left\{\begin{array} { l } 
{ \vec { O D } + \vec { D E } + \vec { E A } = \vec { O A } } \\
{ \vec { O B } + \vec { B H } + \vec { H G } = \vec { O C } + \vec { C G } } \\
{ \vec { O K } + \vec { K J } + \vec { J I } = \vec { O B } + \vec { B I } }
\end{array} \Rightarrow \left\{\begin{array}{l}
l_{1} \cos \theta_{1}+l_{2} \cos \varphi_{1}+l_{3} \cos \varphi_{3}-l_{4} \cos \varphi_{4}=0 \\
l_{1} \sin \theta_{1}+l_{2} \sin \varphi_{1}+l_{3} \sin \varphi_{3}-l_{4} \sin \varphi_{4}=0 \\
l_{6} \cos \varphi_{5}+l_{11} \cos \varphi_{8}+l_{10} \cos \varphi_{7}-l_{5} \cos \varphi_{1}-l_{9} \cos \varphi_{6}=0 \\
l_{6} \sin \varphi_{5}+l_{11} \sin \varphi_{8}+l_{10} \sin \varphi_{7}-l_{5} \sin \varphi_{10}-l_{9} \sin \varphi_{6}=0 \\
l_{8} \cos \theta_{2}+l_{14} \cos \varphi_{2}+l_{13} \cos \varphi_{11}-l_{6} \cos \varphi_{5}-l_{12} \cos \varphi_{9}=0 \\
l_{8} \sin \theta_{2}+l_{14} \sin \varphi_{2}+l_{13} \sin \varphi_{11}-l_{6} \sin \varphi_{5}-l_{12} \sin \varphi_{9}=0
\end{array}\right.\right.
$$

The angular displacement of two active rods $\varphi_{1}, \varphi_{2}$ can be obtained by solving the upper formula:

$$
\left\{\begin{array}{l}
\varphi_{1}=2 \operatorname{acr} \tan \left(\frac{A+\sqrt{B^{2}+A^{2}-C^{2}}}{B-C}\right) \\
\varphi_{2}=2 \operatorname{acr} \tan \left(\frac{D-\sqrt{E^{2}+D^{2}-F^{2}}}{E-F}\right)
\end{array}\right.
$$

The parameters $A, B, C, D, E$ and $F$ can be obtained by formula (1).

The angular velocity of each component can be obtained by calculating the derivative of time to the angular displacement. By the derivative of the formula about the derivative of time, and solving the equation set, the motion law of the two driving level can be obtained:

$$
\left\{\begin{array}{l}
\omega_{1}=\frac{\omega_{4} l_{4} \sin \left(\varphi_{4}-\varphi_{3}\right)}{l_{2} \sin \left(\varphi_{1}-\varphi_{3}\right)} \\
\omega_{2}=\frac{\omega_{4} l_{6} \sin \left(\varphi_{4}+\theta_{3}-\varphi_{11}\right)+\omega_{8} l_{12} \sin \left(\varphi_{8}+\theta_{4}-\varphi_{11}\right)}{l_{14} \sin \left(\varphi_{2}-\varphi_{11}\right)}
\end{array}\right.
$$

\section{Numerical analysis and experiment}

In order to obtain the motion law of two active rods, so as to realize the computer programming control and ensure that the new type of controllable loading mechanism can meet the operating requirements of each typical working condition, after knowing the scale parameters of the controllable loading mechanism components and determining the bucket and boom performance indexes, the inverse kinematics model of the controllable loading mechanism was simulated by MATLAB, and simulation results shown in figure 3 , figure 4 . 


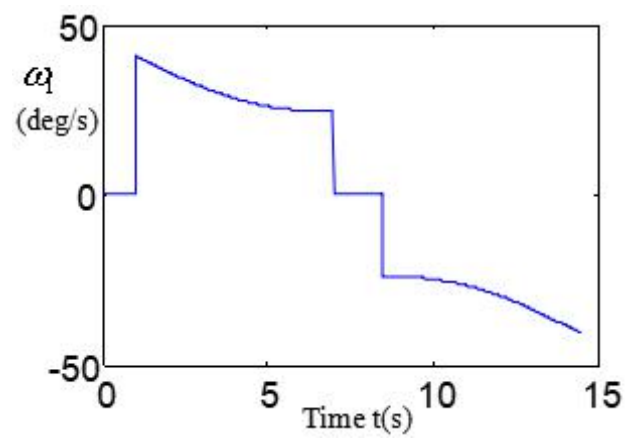

Fig. 3. The change law of the angular velocity of the first driving level.

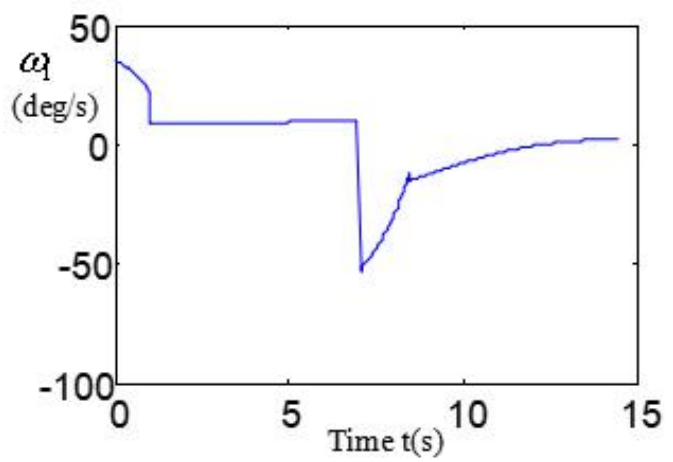

Fig. 4. The change law of the angular velocity of the second driving level.

Through the numerical simulation of MATLAB, the driving parameters that meet the performance requirements of the controllable loading mechanism are obtained. In addition, it can be seen through the simulation that the controllable loading mechanism can control and adjust the output movement of the bucket and boom compared with the conventional loader working device, and the movement regulation can be controlled by the programming of the controllable motor, and the working process is more precise, steady. After the numerical simulation and the subsequent virtual prototyping simulation, a small prototype of the controllable loading mechanism was constructed, as shown in figure 5, figure 6 . The results show that this kind of controllable device can well perform all kinds of working conditions and meet the requirements of loader performance.

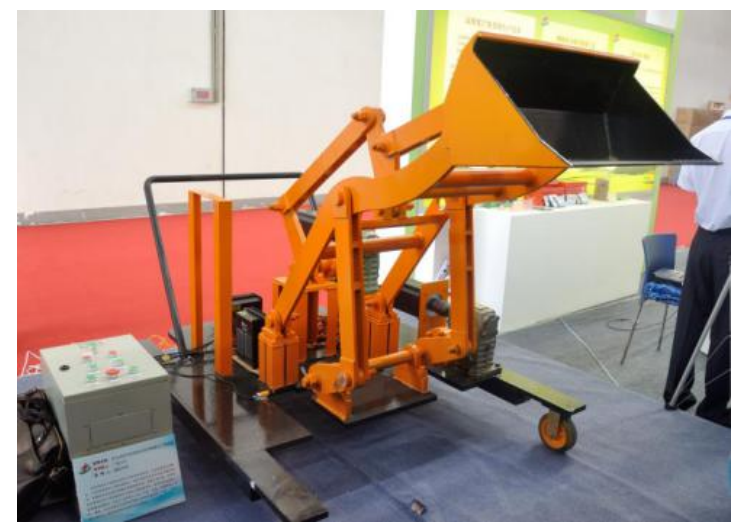

Fig. 5. Small prototype controllable loading mechanism. 


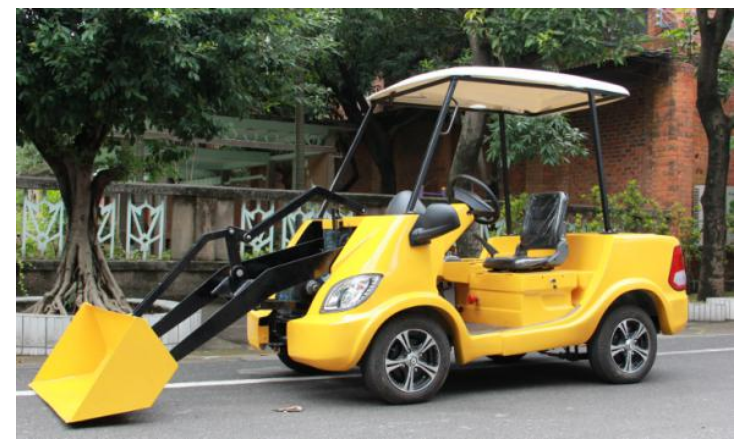

Fig. 6. Small controllable mechanism loader.

\section{Conclusion}

In this paper, the closed-loop vector method is used to establish the inverse kinematics model of a nine-bar 2-DOF controllable loading mechanism. Then, according to the loader performance index, based on the inverse kinematics model of the controllable loading mechanism, the motion law of the two active rods is obtained by using MATLAB numerical calculation, which provides the theoretical basis for the programming control and further research of this type of loading mechanism. Through the establishment of a small prototype model verifies the controllability and feasibility of the controllable loading mechanism, which provides a foundation for further application in the field of microloaders.

\section{References}

1. Paul J, Heney, Kathleen A. Franzinger 2005 Fluid power 20/20 Hydraulics \& Pneumatics, no 1, pp 20-26

2. Zimmerman J, Pelosi M, Williamson C, Ivantysynova M. 2007 Energy Consumption of an LS Excavator Hydraulic System ASME International Mechanical Engineering Congress and Exposition, Ssattle, WA, USA, pp 117-126

3. Paul J, Heney 2005 The big speak out on environmentally friendly systems Hydraulics \& Pneumatics, no 5, pp 26-28

4. Chuenchom T, Kota S 1997 Synthesis of programmable mechanisms using adjustable dyads ASME Journal of Mechanical Design, no 02, pp 232-237

5. Hong B, Erdman A G 2005 A method for adjustable planar and spherical four-bar linkage synthesis Journal of Mechanical Design, vol 127, no 3, pp 456-463

6. Renchung Soong 2010 A new design method for single DOF mechanical presses with variable speeds and length-adjustable driving links Mechanism and Machine Theory, vol 45, no 3, pp 496-510

7. Hongsen Yan, Guojhih Yan 2009 Integrated control and mechanism design for the variable input-speed servo four-bar linkages Mechatronics, vol 19, no 2, pp 174-285

8. Chinedum Okwudire, Jason Rodgers 2013 Design and control of a novel hybrid feed drive for high performance and energy efficient machining CIRP Annals Manufacturing Technology, vol 62, no 1, pp 391-394 
9. R. Whalley, M. Ebrahimi, A.A. Abdul-Ameer 2005 Hybrid modelling of machine tool axis drives International Journal of Machine Tools and Manufacture vol 45, no 14, pp $1560-1576$

10. P.R. Ouyang, Q. Li, W.J. Zhang, L.S. Guo 2004 Design, modeling and control of a hybrid machine system Mechatronics, vol 14, no 10, pp 1197-1217

11. Zhang Lin, Cai Ganwei, Zhu Kaijun, Li Junming, Wang Xiaochun 2017 Kinematic analysis and simulation of the controllable loading mechanism Journal of Machine Design, vol 2, no 2, pp 7-11

12. Zhang Lin, Cai Ganwei, Zhu Kaijun, Li Junming, Wang Xiaochun 2016 Configuration design and applications of controllable loader mechanism based on graph theory China Mechanical Engineering, vol 27, no 4, pp 488-502

13. Luck K. 1994 Computer-aided mechanism synthesis solutions based on the Burmester theory Mechanism and Machine Theory, vol 29, no 6, pp 877-886

14. Fang Xinguo, Zou Huijun, Liang Qinghua, Guo Weizhong 2003 Singularity analysis of 2-DOF planar seven-bar hybrid mechanisms Journal of Shanghai Jiaotong University, vol 37, no 5, pp 674-678

15. Lu Yi, Tatu Leinonen 2002 Computer simulation of approximate dimensional synthesis with four-bar linkage Journal of Computer-Aided Design\&Computer Graphics vol 14, no 6, pp 547-552

16. Lu Yi, Tatu Leinonen 2002 CAD geometric approach for higher-order and combined point-order approximate synthesis Journal of Computer-Aided Design\&Computer Graphics. Vol 14, no 8, pp 772-777 